\title{
A review of Tumor Treating Fields and their future implication in treatment of platinum resistant ovarian cancer
}

\begin{abstract}
Ovarian cancer is a leading cause of morbidity and mortality amongst gynecologic malignancies. Due to the lack of screening tests and early detection, most cases of ovarian cancer are diagnosed in advanced stages. Treatment of patients includes multidisciplinary approaches that include surgical debulking and chemoradiation therapy. Recently, there have been emerging clinical trials that are investigating the use of Tumor treating fields (TTFields) in the treatment of patients with ovarian cancer. Tumor treating fields (TTFields) are a novel, non-invasive cancer treatment modality targeted towards inhibition of solid tumor growth. ${ }^{1,2}$ The use of TTFields was initially approved by the US Food and Drug Administration (FDA) for treatment of recurrent glioblastoma multiforme. In this article, we will be providing an overview of TTFields, including its mechanism of action, burgeoning application in the management of solid tumors, and promising potential in the treatment of patients with platinum resistant ovarian cancer
\end{abstract}

Keywords: tumor treating fields, ovarian cancer, morbidity and mortality, gynecologic malignancies
Volume 12 Issue 2 - 202 I

\author{
Jino Park,' Steven A Toms, ${ }^{2}$ Alexis Svokos ${ }^{3}$ \\ 'Geisinger Commonwealth School of Medicine, USA \\ ${ }^{2}$ Lifespan Health System, Warren Alpert Medical School of \\ Brown University, USA \\ ${ }^{3}$ Department of Obstetrics and Gynecology, Geisinger Medical \\ Center, USA
}

\section{Correspondence: Alexis Svokos MD, Geisinger Medical Center, Dept of Obstetrics and Gynecology, 100 N Academy Ave, Danville PA I782I, USA, Tel 570-27I-6343,}

Email asvokos@geisinger.edu

Received: March 18, 2021 | Published: March 29, 202 |
Abbreviations: TTFields, tumor treating fields; FDA, food and drug administration; PFI, platinum free interval; GBM, glioblastoma; NSCLC, non-small cell lung cancer; GBM, glioblastoma multiforme; PFS, progression free survival; OS, overall survival; RECIST, response evaluation criteria in solid tumors

\section{Introduction}

Worldwide, ovarian cancer is the second most common type and the leading cause of death amongst gynecologic malignancies. ${ }^{3}$ Due to lack of symptoms in early stages of disease and no standardized screening tests, the majority of the cases are diagnosed in advanced Stage III or Stage IV. ${ }^{4}$ Standard fist-line treatment involves surgical debulking, radiation and combination chemotherapy with platinum and taxane agents. ${ }^{3}$ Platinum works by forming crosslinks between purine bases of DNA which in turn disrupt DNA repair and cause DNA damage, ultimately leading to apoptosis of cancer cells. ${ }^{5}$ In addition to bone marrow suppression and gastrointestinal disturbances, side effects of platinum include ototoxicity, renal dysfunction, and peripheral neuropathy. Taxanes on the other hand, target microtubules to arrest cell division in the G2 or M phase. They exert cytostatic effects by disrupting spindle microtubule dynamics and are known for neurotoxicity.

A significant portion of patients that undergo primary treatment achieve complete remission; however, disease recurrence occurs in up to $80 \%$ of patients. The prognosis of patients with platinum resistant ovarian cancer is even worse, with a 5 -year survival rate of approximately $27.4 \%$ in patients with metastatic disease. ${ }^{6}$ The management of recurrent disease, all of which eventually displays platinum resistance, is dependent upon the time elapsed between completion of platinum-based chemotherapy and recurrence, referred to as platinum free interval (PFI). PFI was defined during the 2010 Ovarian Cancer Consensus Conference as the interval between the date of last treatment of platinum therapy and the date of relapse detection. $^{?}$

Patients with PFI of less than one month are considered platinum refractory, while patients with PFI of one to six months are considered platinum resistant. Despite remarkable progress in the management of epithelial ovarian cancer, the treatment of patients with platinum refractory and resistant disease remains a challenge. Typically, treatment of these patients involves weekly paclitaxel administration, as a single agent or in combination with bevacizumab. ${ }^{8,9}$ For patients who previously had disease progression on paclitaxel or are not proper candidates for paclitaxel therapy, pegylated liposomal doxorubicin remains a treatment option; however, response rates are below $20 \% .{ }^{10}$ Alternative treatment options such as topotecan and gemcitabine also associated with overall low response rates. ${ }^{11,12}$

Hence, the limited improvement of overall survival rates by various combinations of chemotherapy calls for new strategies and treatment modalities.

Recently, there has been rising interest in treatment of patients with recurrent epithelial ovarian, fallopian tube, or primary peritoneal cancer with TTFields as an additive therapy to standard surgical and chemoradiative interventions to provide synergistic antitumoral effects.

\section{Physics and cell biology processes utilized by Tumor Treating Fields on disruption of mitotic tumor cells}

The development of Tumor treating fields (TTFields) by Dr. Yorum Palti at the Rappaport Institute in Israel is based on the well-established physical properties of dipole alignment and dielectrophoresis. TTFields therapy is a non-invasive procedure targeted towards treatment of solid tumors. 
The biophysics behind TTFields is based on the concept that living cells are made of charged molecules and ions, which therefore respond to electrical fields and currents. This concept also applies to many key biological processes including cell division. The effect depends on the magnitude of the electrical potential difference between the two electrodes (field intensity) and the frequency. At very low frequencies $(<1 \mathrm{kHz})$, excitable cells such as neurons or myocytes will be depolarized. ${ }^{13}$ At very high frequencies $(>\mathrm{MHz})$, heat is generated in the tissues due to dielectric loss. For instance, cardiac pacemakers and deep brain stimulators work in a low frequency range. High frequency range is used in radiofrequency ablations or diathermy treatments. ${ }^{13}$ Intermediate alternating frequencies of electrical fields (range, $10-1000 \mathrm{kHz}$ ) is too fast to induce cell depolarization and too slow to generate any significant heat. Instead, intermediate electrical field frequencies have been noted to influence biological tissues by microscopic particle alignment, cell rotation, and transient pore formation in cell membranes. ${ }^{14}$

Tumor treating fields (TTFields) are alternating, low-intensity, intermediate frequency electric fields that disrupt cell division and inhibit tumor growth via apoptosis. TTFields and paclitaxel have a similar mechanism of action in that they target tubulin and are regarded as anti-spindle therapy. ${ }^{15}$ In addition, TTFields seem to perturb cells at the transition from metaphase to anaphase and cells exposed to the TTFields during mitosis exhibited membrane blebbing and progress to apoptosis coinciding with metaphase exit, similar to paclitaxel. ${ }^{16}$ TTFields also may increase membrane permeability in glioblastoma cells. ${ }^{17}$

However, TTFields differ from paclitaxel in that they increase the amount of non-polymerized tubulin in cells. Initial in vitro research revealed that TTFields exerted a significant growth inhibitory effect on a variety of quickly dividing tumor cell lines by causing cell cycle arrest and apoptosis without affecting non-dividing cells. ${ }^{18}$ These results were later confirmed in rabbit and mice tumor models. ${ }^{19}$ Further studies demonstrated that interference on the mitotic spindle apparatus is key to the growth inhibitory effect. ${ }^{13}$ TTFields target proteins with large dipole moments such as the spindle microtubules which play a crucial role in the metaphase and anaphase stages of mitosis for separation and equal distribution of chromosomes. ${ }^{14}$ By inhibiting the polymerization of microtubules, the mitotic spindle apparatus cannot properly assemble. In telophase, the dielectrophoretic forces induced by the electric fields compromise normal cytokinesis. ${ }^{18}$ The disruption of the normal cell division process, thereby hindering cell proliferation, is the foundation by which TTFields exert antitumoral effects. ${ }^{20}$

Animal models of tumors such as glioblastoma (GBM), non-small cell lung cancer (NSCLC), melanoma, and pancreatic cancer showed that application of TTFields at the appropriate frequencies, inhibited tumor growth and metastatic spread. ${ }^{13}$ Rat models inoculated with GBM cells were treated with TTFields and showed a decreased size of tumors compared with untreated rats. ${ }^{21}$ A synergistic effect was seen TTFields were used along with chemotherapy agents such as paclitaxel, doxorubicin and cyclophosphamide. ${ }^{21}$ There has been some speculation that TTFields may also impact immune system response to tumors. There is some preliminary in vitro evidence to support this notion by suggesting macrophage activation by TTFields. ${ }^{22}$

In summary, TTFields disrupt the mitotic cell cycle during metaphase, anaphase, and telophase. These results in cell cycle arrest or delay in cell division and interfere with the assembly of the spindle apparatus resulting in unequal chromosome distribution. The cells cannot divide and die in apoptosis. For the best treatment effect, the electric field intensity and frequency is adapted to the tumor type and cell size. To maximize the beneficial antitumor effect, the frequency used is inversely correlated with cell size. ${ }^{19}$ Prolonged exposure to the electrical fields is required for maximal effect because cell division may take place anytime. In fact, there is clinical evidence that both density and treatment compliance may impact the response to TTFields. ${ }^{23,24}$ TTFields were initially studied in clinical trials of patients with Glioblastoma Multiforme (GBM). Currently, TTField use has been approved by US FDA for treatment of GBM and mesothelioma. ${ }^{25}$

In patients treated with maintenance temozolomide concurrent TTField use increased the overall 5-year survival rate from 5\% to $13 \%$, without any evidence of systemic toxicity. ${ }^{26}$ The large EF-11 phase III multicenter clinic trial compared overall survival in patients with recurrent glioblastoma undergoing TTFields treatment alone versus standard chemotherapy. ${ }^{27}$ Median survival rates amongst the two groups were comparable (6.6 months in patients treated with TTFields vs 6.0 months in patients treated with chemotherapy), and 1 year survival rate was $20 \%$ amongst both groups. Progression free survival rate at 6 months at was $21.4 \%$ in patients treated with TTFields vs $15 \%$ in the standard chemotherapy group ${ }^{27}$ In clinical use for patients with GBM, the delivery of TTFields is achieved with a portable and battery-powered device. The electric field is delivered to the brain via four transducer arrays with insulated electrodes and continuous temperature sensing fixed to the patient's shaved scalp. Therapy delivers continuous, low intensity $(1-3 \mathrm{~V} / \mathrm{cm})$, intermediate frequency $(200 \mathrm{kHZ})$, alternating electric fields to the tumor region. ${ }^{1,2}$ The electric fields produced via the paired transducer arrays applied directly on the skin surface, interfere with the mitotic process of cancerous cells by inhibiting the normal polymerization process of the mitotic spindle during metaphase leading to mitotic arrest and cancer cell death. ${ }^{13}$

Currently, there are several ongoing phase 3 clinical trials investigating TTField use in patients with non-small cell lung cancer, pancreatic cancer, brain metastasis secondary to non-small cell lung cancer and ovarian cancer. ${ }^{28}$

\section{Future implications of TTFields use in the treatment of patients with platinum resistant ovarian cancer}

Current guidelines for chemotherapy treatment of both optimally as well as sub-optimally debulked ovarian cancer patients involve combination of a platinum agent (cisplatin or carboplatin) and paclitaxel. ${ }^{29}$ Additionally, weekly paclitaxel administration, as a single agent or in combination with bevacizumab ${ }^{8,9}$ is the treatment choice for patients with platinum resistant or refractory disease.

A recent study by Voloshin et al investigated the effects of treatment with TTFields alone and in combination with paclitaxel in ovarian cancer cells in vitro and in vivo. ${ }^{30}$ Application of TTFields in vitro showed significant inhibitory effect on the viability of human ovarian cancer cells..$^{30}$ In vitro studies of ovarian cancer cells with combinations of TTFields and paclitaxel, revealed that relative to paclitaxel alone, combination treatment significantly increased cell apoptosis estimated by cell count by synergistically or additively enhancing the effects of paclitaxel on three different ovarian cell 
lines. ${ }^{30}$ When studied in vivo using cells that resemble human ovarian cancer orthotopically implanted into the ovarian bursa of murine models, it was shown that while TTFields alone did not reduce tumor weight and volume, combination treatment yielded significant reductions in both weight and volume compared to untreated control mice or those treated with either paclitaxel or TTFields.

The INNOVATE (EF-22) trial is the first clinical trial that investigated the safety and efficacy of using TTFields in conjunction with weekly administration in 31 patients with platinum resistant ovarian cancer. ${ }^{31}$ Enrolled patients were heavily pre-treated with surgery, radiation, and chemotherapy but relatively uncomplicated with an ECOG score of 0-1. All had histologically confirmed recurrent epithelial ovarian, uterine tube, or primary peritoneal carcinoma. A treatment regimen optimized by the previously mentioned in vivo studies by Voloshin were applied, including TTFields frequency of $200 \mathrm{kHz}$ and maximal amplitude of $114 \mathrm{~mA}$ transduced through two pairs of arrays directly adhered to the skin of the abdomen, back and pelvis. Concomitant intravenous administration of $80 \mathrm{mg} / \mathrm{m}^{2}$ of paclitaxel were given once every seven days. On average, patients received treatments with TTFields for 14 hours a day for 17 weeks and paclitaxel for 22 weeks. On average, patients received treatments with TTFields for 14 hours a day, for 17 weeks and paclitaxel for 22 weeks. The daily duration of treatment with TTFields in this study was lower than in the EF14 trial where the daily duration of TTField treatment in Glioblastoma Multiforme patients was a minimum of 18 hours. ${ }^{26}$

Both safety and efficacy profiles from the INNOVATE study displayed promising results. While nearly half of the patients experienced some sort of gastrointestinal symptoms, fatigue, edema, or neuropathy, these side effects were mild in severity and attributable to concomitant use of paclitaxel or the malignancy itself. Contact dermatitis, the expected adverse effect of TTFields, was present in $93 \%$ of patients, but was readily managed with topical corticosteroids and maintenance of good skin hygiene which helped limit the number of severe, grade 3 dermatitis to only two patients.

Prior secondary analysis that investigated quality of life outcomes of patients with Glioblastoma who were being treated with TTFields reported similar findings, with skin reaction and itchiness being the most experienced adverse reaction. ${ }^{32}$ When comparing the group of patients that were treated with TTFields versus those that did not, there was no significant difference in the patient's health-related quality of life (HRQoL) except for dermatitis reaction. ${ }^{32}$

The efficacy of the combination of TTFields and paclitaxel were measured by progression free survival (PFS), overall survival (OS), and per The Response Evaluation Criteria in Solid Tumors (RECIST) 1.1. Median PFS was reported to be 8.9 months, OS at 6 months $90 \%$ and at 12 months $61 \%$, and $71 \%$ of patients were determined to have gained clinical benefit per RECIST. As reference, meta-analysis of 1640 patients with recurrent ovarian cancer on primary platinum and taxane therapy demonstrated PFS of 4.4 months at their 4th relapse and median OS of 6.2 months. ${ }^{33}$ Another study showed similar patients treated with weekly paclitaxel alone had PFS of 5.4 months. ${ }^{34}$ PFS 6.7 months were reported or patients with platinum-resistant ovarian cancer treated with bevacizumab. More research is warranted to elucidate the full potential of TTFields in the treatment of ovarian cancer as well as other treatment-resistant neoplastic diseases after these pilot studies revealed such favorable results. The INNOVATE phase 2 trial has shown less severe adverse effects, improved survival, and significant clinical benefit to be gained from TTFields that can be life-changing for the cancer patient who has failed first-line treatment. ${ }^{35}$

\section{Conclusion and future directions}

Ovarian cancer is the leading cause of death amongst gynecologic malignancies. Despite multidisciplinary approaches to treatment of patients, that include surgical debulking and chemoradiation therapy, the overall 5-year survival rate is low. Newest studies have profound impact on the use of TTFields in the treatment of patients with platinum resistant ovarian cancer. Preliminary data are promising in improving the overall response and survival data with minimal side effects. A phase 3 randomized trials is currently in progress.

\section{Acknowledgments}

None.

\section{Funding}

None.

\section{Conflicts of interest}

The author declares that there is no conflict of interest regarding this study.

\section{References}

1. Kirson ED, Gurvich Z, Schneiderman R, et al. Disruption of cancer cell replication by alternating electric fields. Cancer Res. 2004;64:3288-3295.

2. Kirson ED, Dbalý V, Tovarys F, et al. Alternating electric fields arrest cell proliferation in animal tumor models and human brain tumors. Proc Natl Acad Sci USA. 2007;104:10152-10157.

3. Ferlay J, Soerjomataram I, Dikshit R, et al. Cancer incidence and mortality worldwide: sources, methods and major patterns in GLOBOCAN 2012. Int J Cancer. 2015;136(5):E359-E386.

4. Jelovac D, Armstrong DK. Recent progress in the diagnosis and treatment of ovarian cancer. Cancer J Clin. 2011;61:183-203.

5. Dasari S, Tchounwou PB. Cisplatin in cancer therapy: molecular mechanisms of action. Eur J Pharmacol. 2014;740:364-378.

6. Markman M, Markman J, Webster K, et al. Duration of response to second-line, platinum based chemotherapy for ovarian cancer: implications for patient management and clinical trial design. $J$ Clin Oncol. 2004;22:3120-125.

7. Friedlander M, Trimble E, Tinker A, et al. Clinical trials in recurrent ovarian cancer. Int J Gynecol Cancer. 2011;21:771-775.

8. Seewaldt VL, Greer BE, Cain JM, et al. Paclitaxel (Taxol) Treatment for refractory ovarian cancer: phase II clinical trial. Am J Obstet Gynecol. 1994;170:1666-1670.

9. Gore ME, Levy V, Rustin G, et al. Paclitaxel (Taxol) in relapsed and refractory ovarian cancer: the UK and Eire experience. $\mathrm{Br} J$ Cancer. 1995;72:1016-1019.

10. Gordon AN, Fleagle JT, Guthrie D, et al. Recurrent epithelial ovarian carcinoma: a randomized phase III study of pegylated liposomal doxorubicin versus topotecan. J Clin Oncol. 2001;19:3312-3322.

11. Mutch DG, Orlando M, Goss T, et al. Randomized phase III trial of gemcitabine compared with pegylated liposomal doxorubicin in patients with platinum-resistant ovarian cancer. J Clin Oncol. 2007;25:2811-2818. 
12. Sehouli J, Stengel D, Harter P, et al. Topotecan weekly versus conventional 5 day schedule in patients with platinum-resistant ovarian Cancer: a randomized multicenter phase II trial of the north-eastern German society of gynecological oncology ovarian cancer study group. J Clin Oncol. 2011;29:242-248.

13. Hottinger AF, Pacheco P, Stupp R. Tumor treating fields: a novel treatment modality and its use in brain tumors. Neuro Oncol. 2016;18(10):13381349.

14. Kirson ED, Dbaly V, Tovarys F, et al. Alternating electric fields arrest cell proliferation in animal tumor models and human brain tumors. Proc Natl Acad Sci USA. 2007;104(24):10152-10157.

15. Davies AM, Weinberg U, Palti Y. Tumor treating fields: a new frontier in cancer therapy. Ann NY Acad Sci. 2013;1291:86-95.

16. Gera, N, Yang A, Holtzman TS, et al. Tumor treating fields perturb the localization of septins and cause aberrant mitotic exit. 2015;10(5):0125269.

17. Chang E, Patel CB, Pohling C, et al. Tumor treating fields increases membrane permeability in glioblastoma cells. Cell Death Discovery. 2018:4:113.

18. Storm FK, Morton DL, Kaiser LR, et al. Clinical radiofrequency hyperthermia: a review. Natl Cancer Inst Monogr. 1982;61:343-350.

19. Kirson ED, Giladi M, Gurvich Z, et al. Alternating electric fields (TTFields) inhibit metastatic spread of solid tumors to the lungs. Clin Exp Metastasis. 2009;26(7):633-640.

20. Giladi M, Schneiderman RS, Voloshin T, et al. Mitotic spindle disruption by alternating electric fields leads to improper chromosome segregation and mitotic catastrophe in cancer cells. Sci Rep. 2015;5:18046.

21. Kirson ED, Dbaly V, Tovarys F, et al. Alternating electric fields arrest cell proliferation in animal tumor models and human brain tumors. Proc Natl Acad Sci USA. 2007;104(24):10152-10157.

22. Park J-I, Song K-H, Jung S-Y, et al. Tumor-treating fields induce RAW264.7 macrophage activation via NK-кB/MAPK signaling pathways. Technology in Cancer Research \& Treatment. 2019.

23. Ram Z, Kim CY, Nicholas G, et al. Increased compliance with Tumor Treating Fields (TTFields) therapy is prognostic for improved survival in the treatment of glioblastoma: A subgroup analysis of the EF-14 Phase III trial. J Neurooncol. 2019;141(2):467-473.

24. Taphoorn MJB, Dirven L, Kanner AA, et al. Influence of treatment with tumor-treating fields on health-related quality of life of patients with newly diagnosed glioblastoma: a secondary analysis of a randomized clinical trial. JAMA Oncol. 2018;4(4):495-504.
25. Ceresoli GL, Aerts JG, Dziadziuszko R, et al. Tumour Treating Fields in combination with pemetrexed and cisplatin or carboplatin as first line treatment for unresectable malignant pleural mesothelioma (STELLAR): a multicentre, single-arm phase 2 trial. Lancet Oncol. 2019;20(12):17021709

26. Stupp R, Taillibert S, Kanner A, et al. Effect of tumor-treating fields plus maintenance temozolomide vs maintenance temozolomide alone on survival in patients with glioblastoma: a randomized clinical trial. JAMA. 2017;318(23):2306-2316.

27. Stupp R, Wong ET, Kanner AA, et al. Novo TTD-100A versus physician's choice chemotherapy in recurrent glioblastoma: a randomized phase III trial of a novel treatment modality. Eur J Cancer. 2012;48:2192-2202.

28. Ongoing Tumor Treating Fields clinical trials for cancer registered in Clinical Trials.gov. 2020

29. Sandercock J, Parmar MK, Torri V, et al. First line treatment for advanced ovarian cancer: paclitaxel, platinum and the evidence. $\mathrm{Br} J$ Cancer. 2002;87:815-824

30. Voloshin T, Munster M, Blatt R, et al. Alternating electric fields (TTFields) in combination with paclitaxel are therapeutically effective against ovarian cancer cells in vitro and in vivo. Int J Cancer. 2016;139:2850-2858.

31. Vergote I, von Moos R, Manso L, et al. Tumor treating fields in combination with paclitaxel in recurrent ovarian carcinoma: Results of the INNOVATE pilot study. Gynecol Oncol. 2018;150:471-477.

32. Stupp R, Taillibert S, Kanner A, et al. Effect of tumor-treating fields plus maintenance temozolomide vs maintenance temozolomide alone on survival in patients with glioblastoma: a randomized clinical trial. JAMA. 2017;318(23):2306-2316.

33. Hanker L, Loibl S, Burchardi N, et al. The impact of second to sixth line therapy on survival of relapsed ovarian cancer after primary taxane/ platinum-based therapy. Ann Oncol. 2012;23:2605-2612.

34. Monk B, Poveda A, Vergote I, et al. Anti-angiopoietin therapy with trebananib for recurrent ovarian cancer (TRINOVA-1): a randomised, multicentre, double-blind, placebo-controlled phase 3 trial. Lancet Oncol. 2014;15:799-808.

35. Poveda A, Selle F, Hilpert F, et al. Bevacizumab combined with weekly paclitaxel, pegylated liposomal doxorubicin, or topotecan in platinumresistant recurrent ovarian cancer: analysis by chemotherapy cohort of the randomized phase III AURELIA trial. J Clin Oncol. 2015;33:3836-3838. 